\title{
Direct Processing of a Flow Reaction Mixture Using Continuous Mixed Suspension Mixed Product Removal Crystallizer
}

\author{
Kornélia Tacsi, Hajnalka Pataki,* András Domokos, Brigitta Nagy, István Csontos, Imre Markovits, \\ Ferenc Farkas, Zsombor Kristóf Nagy, and György Marosi
}

Cite This: Cryst. Growth Des. 2020, 20, 4433-4442

Read Online

\section{ACCESS \\ Џlll Metrics \& More \\ Article Recommendations \\ Supporting Information}

ABSTRACT: Crystallization as the most widespread purification, separation, and morphology-determining method is a critical technology that could be made safer and more economical by using continuous crystallization alternatives. Accordingly, this study aims to develop the continuous crystallization method for direct processing of a flow reaction mixture of acetylsalicylic acid (ASA) and to provide pure, homogeneous crystalline products for further formulation steps. The solid-liquid separation and the purification of the acetylsalicylic acid from the multicomponent mixture were accomplished in a single stage mixed suspension mixed product removal (MSMPR) continuous crystallizer equipped with an overflow and an inner buffer element to ensure

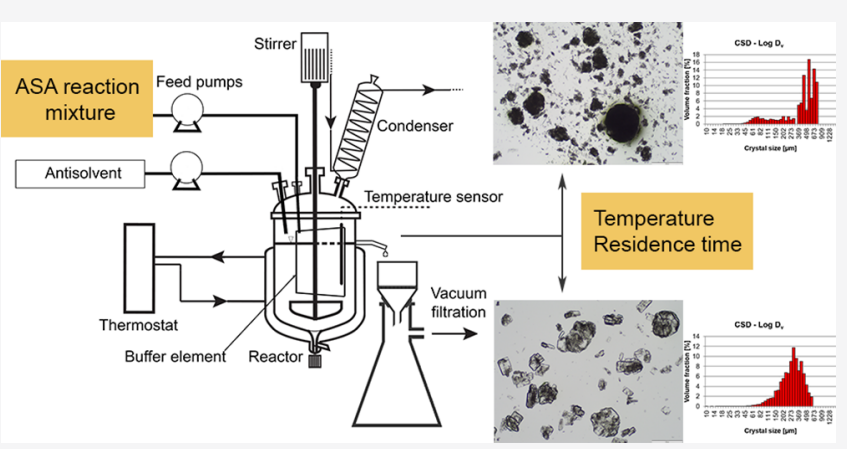
the representative withdrawal of the product suspension. The effect of process parameters such as the operating temperature and the length of residence time (RT) on product quality and quantity were studied at two and three levels, respectively. Investigating these parameters, we found that higher operating temperatures $\left(25^{\circ} \mathrm{C}\right)$ and longer residence time $(47$ min) favor appropriate purity $(>99.5 \%)$, and narrow crystal size distribution. By reducing the operating temperature $\left(2.5{ }^{\circ} \mathrm{C}\right)$, the yield improved slightly (approximately 77\%) and polydisperse products were characterized. The developed crystallization process can link the flow synthesis with the continuous formulation, and consequently serves a further step toward end-to-end production.

\section{INTRODUCTION}

Changing of batch processes to continuous technologies has become an urgent issue in the pharmaceutical industry since the early 2000s. Batch manufacturing is widespread, while using continuous technologies is on the elementary stage compared to other fields with the same production volume. ${ }^{1}$ The slow conversion to continuous techniques could be explained by the complexity of the procedures and the former conservative regulating attitude of the authorities, among other causes. In recent years, recognizing the many advantages of continuous manufacturing, the regulatory bodies in cooperation with the pharmaceutical companies have been urging the conversion to continuous processes. ${ }^{2-4}$ Several synthetic and formulation procedures have been accomplished in a continuous mode; however, only a few studies have dealt with the direct connection of these steps. ${ }^{5,6}$ Thus, linking the flow synthesis with the continuous formulation procedures using continuous crystallization technology (for direct isolation and purification of the flow reaction mixture) is a remarkable stage toward end-to-end manufacturing.

The quality of products manufactured by conventionally used batch processes can change from batch to batch. ${ }^{7}$ In contrast, by implementing the continuous crystallization methods, the production of solid active pharmaceutical ingredients (APIs) could be accomplished in steady state using a much smaller size of equipment. Choosing the right parameters, the quality and quantity of the crystals could be affected, and thus it makes the product features designed. Therefore, in many cases, the production chain is shortened by the elimination of various formulation processes. All of these differences result in a decrease in investment costs by approximately $20 \% .^{3}$

The kinetics of crystallization determines the continuous crystallization technique and equipment to choose. ${ }^{7,8}$ Processes with slower conversion require longer residential time, which could be accomplished mainly in a continuous stirred tank crystallizer. The most common type of continuous tank crystallizer is the mixed suspension mixed product removal (MSMPR) reactor system, ${ }^{9,10}$ which could be operated in a single stage or in multiple stages. If the crystallization kinetics is fast, then plug flow crystallizers ${ }^{11}$ can be preferred ensuring

Received: February 26, 2020

Revised: May 21, 2020

Published: May 21, 2020 
efficient mixing and short residence time. ${ }^{7,12,13}$ MSMPR, a key device in the continuous crystallization studies, is practically the same instrument that is utilized in batch processes. ${ }^{4,14,15}$ In parallel, it performs the difficulties attached to batch equipment as well. Previous studies have reported simplicity, lower investment, and maintenance costs ${ }^{7,16}$ as the main advantages of MSMPR crystallizers. Nevertheless, the reasonably longer start-up period, occurrence of product inconsistency (due to nonstable operation), problematic scale-up, and low efficiency are disadvantageous compared to tubular crystallizers.

In a conventionally operating MSMPR reactor system, the product slurry is withdrawn continuously and the product properties are generally the same as the agitated slurry. Usually, peristaltic pumps are used for product removal; however, other withdrawal techniques, such as "intermittent withdrawal", $8,17-20$ are becoming increasingly popular, which prevents the blockage of the transfer lines. Using "intermittent withdrawal", approximately $10 \%$ of the slurry volume is withdrawn in every one-tenth of residence time. Another novel approach, the periodic MSMPR crystallizer ${ }^{21}$ was designed by Powell et al., when no addition or withdrawal to/from the crystallizer was applied between the suspension transfer period, but the agitation remained constant. In that work, the concept of the "state of controlled operation" was investigated rather than "steady-state operation". By implementing periodic slurry transfer, more representative withdrawal of the slurry could be achieved, while the possibility of blockage and encrustation was reduced. These withdrawal techniques usually employ peristaltic pumps ${ }^{22,23}$ or vacuum, ${ }^{8,18,24}$ though the former has a tendency to cause damage to crystals. Other ways of continuous suspension withdrawal, overflow techniques are presented just in a few publications using vertical ${ }^{25}$ or lateral ${ }^{26,27}$ overflow tubes or pipes. These could help to avoid the clogging of the transfer line and the breaking of crystals, by eliminating the conventionally used peristaltic pumps. As these instruments are different from batch tank reactors, their implementation requires more investments. This could be an explanation of their underrepresentation in the literature.

Most of the continuous crystallizations discussed in the literature started from the clear solution of arbitrary and mostly high drug concentration. ${ }^{18,25,28,29}$ Processing of the steadystate composition of a flow reaction mixture, containing impurity byproducts, is challenging as the number of variable parameters of crystallization is reduced. Just a few researchers are concerned with this issue. For instance, Zhang et al. ${ }^{15}$ published the cooling/antisolvent continuous crystallization of an API in a two-stage cascade MSMPR crystallizer. The feed solution consisted of the API and impurities in the mixture of ethyl acetate and water from a previous separation step, and heptane was chosen as an antisolvent. Though pure materials were used during the experiments, the composition of the feed was the same as in the actual reaction mixture. This continuous crystallization procedure was embedded in the publication of Mascia et al. ${ }^{5}$ about the end-to-end manufacturing of aliskiren hemifumarate. Adamo et al. ${ }^{30}$ also developed a compact, reconfigurable end-to-end system, in which only a semibatch crystallization step was used as the first part of the downstream procedure. In another study, Richardo and Xiongwei ${ }^{31}$ set up a cleaning protocol for vanisal sodium and aspirin production in a continuous oscillatory baffled reactor (COBR). First, the mentioned compounds were produced in one-step chemical reactions in the first segment of the COBR, and then the drugs were purified and separated through cooling crystallization in the remaining baffled pipe sections of the COBR. The main goal was to establish an effective cleaning method during the transition between the production of different APIs and fine chemicals. Accordingly, the yield and purity of the products were characterized without examining the crystal size, habit, and morphology.

Our work aimed to develop a continuous crystallization technique adjusted to the end-to-end production line of acetylsalicylic acid (ASA). For this purpose, the processing of a reaction mixture was investigated using MSMPR crystallizer. To avoid the use of a peristaltic pump at product withdrawal, the crystallizer was equipped with overflow tubing and an internal vertical plate served as a buffer element. It was also required to produce large, homogeneous crystals with desired purity being suitable for direct compression. Additionally, the process parameter dependence of the yield, the product purity, and also the crystal morphology had to be examined in detail. The mixture was produced in a flow reactor according to a method published recently by the authors. ${ }^{6}$ The mixture contained the solvent, catalyzer, starting materials, and secondary products of the synthesis.

\section{EXPERIMENTAL MATERIALS AND METHODS}

2.1. Materials. The solvent composition of the flow reaction mixture can be found in Table 1. Acetylsalicylic acid (ASA, >99\%)

Table 1. Purity, V/V Ratio, and Source of the Solvents in the Reaction Mixture

$\begin{array}{llcl} & \text { purity } & V / V \% & \text { source } \\ \text { Ethyl acetate }(\mathrm{EtOAc}) & & 79.5 & \text { Merck Millipore } \\ \text { Acetic acid }(\mathrm{AcOH}) & 99-100 \% & 16.3 & \\ \text { Ethanol (EtOH) } & \geq 99 \% & 3.8 & \\ \text { Phosphoric acid }\left(\mathrm{H}_{3} \mathrm{PO}_{4}\right) & 85 \text { wt \% } & 0.4 & \end{array}$

and salicylic acid (SA, >99.0\%) were obtained from Sigma-Aldrich. The $n$-heptane $(>96 \%)$ was purchased from Molar Chemicals. The acetonitrile (ACN, >99.9\%) used for the HPLC measurements was obtained from Merck. The concentration of ASA in the reaction mixture was $91.9 \mathrm{mg} / \mathrm{mL}$ or $0.097 \mathrm{~g}$ (ASA)/g (solvent mixture); the SA impurity concentation was $4.84 \mathrm{mg} / \mathrm{mL}$.

The feed solution was prepared by measuring the pure components of the reaction mixture in a defined amount and stirring them until complete dissolution of the solid compounds. Besides ASA, salicylic acid $(<3 \%)$, acetylsalicylic anhydride $(<1 \%)$ were found in a $<5 \%$ amount during flow synthesis. Among the secondary products, salicylic acid (SA) has the highest amount, and thus the impurities are represented by $5 \%$ of SA. Antisolvent and combined cooling/ antisolvent crystallization of the flow reaction mixture was performed in an MSMPR crystallizer as described in the following section.

2.1.1. Continuous Crystallization in the MSMPR Crystallizer. All continuous crystallization experiments were performed in a $250 \mathrm{~mL}$ jacketed glass reactor (DN 60, Schmizo, Schwitzerland) equipped with a Eurostar power control-visc type stirrer (IKA, Germany) and a PTFE coated Ruston 6-blade impeller with $35 \mathrm{~mm}$ overall dimensions (horizontal). To control the temperature, a jacket temperature control was used with a monofluid thermostat (Huber Ministat 230). The temperature of the suspension was measured with a Pt-100 thermometer. The reactor system is illustrated in Figure 1. The withdrawal of the slurry was obtained utilizing an overflow tubing; hence, the discharge of the suspension was continuous and equivalent to the feeding rate. A PTFE vertical plate, used as a buffer element, was also placed in the reactor to separate the feed from the withdrawal, hereby to ensure the uniform residence time distribution 


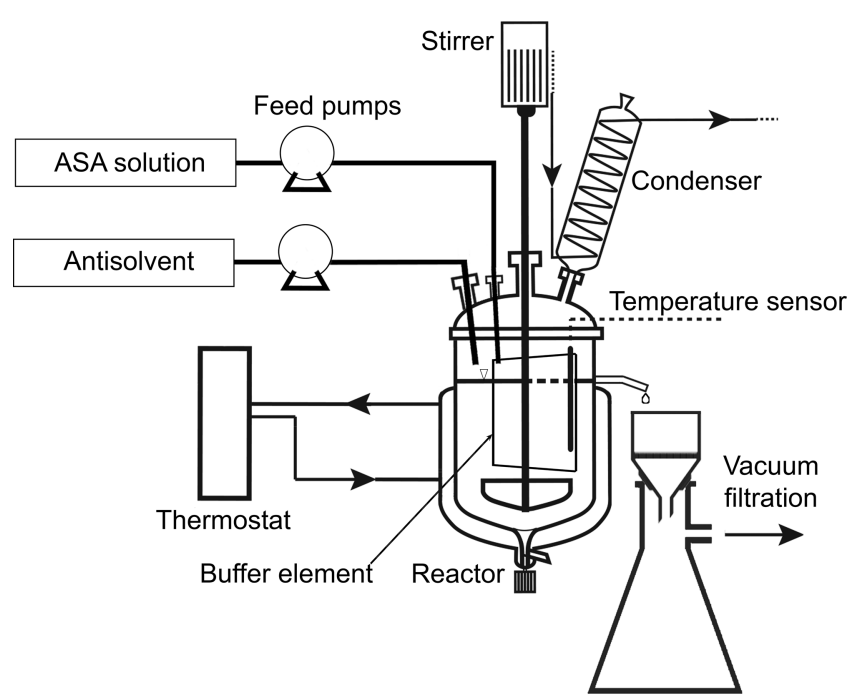

Figure 1. Schematic image of the experimental setup.

of the crystals. The feed streams were dripped on the slurry agitated in the reactor. The crystals had to dive under the plate to reach the overflow tubing. The suspension was agitated with $700 \mathrm{rpm}$ speed to ensure representative or isokinetic withdrawal and avoid sedimentation of the crystals. The jacket temperature was set at 0 or $25{ }^{\circ} \mathrm{C}$ resulting in 2.5 or $25^{\circ} \mathrm{C}$ solution temperature that did not vary during the whole process.

At the beginning of the experiments, $40 \mathrm{~mL}$ heptane was placed in the empty reactor and was tempered at the desired temperature. During agitation, $10 \mathrm{~mL}$ of the reaction mixture was pipetted to the heptane to make a starting suspension. The ASA solvent and antisolvent streams were dripped on this starting suspension. The feed of the reaction mixture was performed with a JASCO PU-980 HPLC pump, and Kappa 10PP single-piston isocratic pumps were used for antisolvent intake. The product slurry flown out through the overflow and the crystals were filtered directly using G2 or G3 glass filters connected to a continuously operating water jet pump. The samples were dried at room temperature without washing the filter cake. During each experiment, we collected product samples at least 12 times for 5 to $20 \mathrm{~min}$, depending on the residence time duration. The experiments lasted for 20 residence times, as we intended to examine the system after reaching steady-state conditions. Therefore, the duration of experiments was 4,8 , or $16 \mathrm{~h}$ depending on the residence time.

From just-filtered wet samples, HPLC samples were produced by dissolving 1-5 $\mathrm{mg}$ of crystals in EtOH to make a $1 \mathrm{mg} / \mathrm{mL}$ concentration solution. This solution was diluted with a mixture of ACN:MeOH: $\mathrm{H}_{3} \mathrm{PO}_{4}$ (85\%) (92:8:0.5 v/v ratio) to reach $1: 20$ dilution. The filters with samples were weighed after drying at room temperature to calculate the yield. The yield calculation was based on the total ASA content $\left(m_{f}, \mathrm{~g}\right)$, that was determined with the following equation:

$$
m_{\mathrm{f}}=t_{\mathrm{f}} \times \dot{V}_{\mathrm{s}} \times c
$$

where $t_{\mathrm{f}}$ is sampling duration in minutes, $\dot{V}_{\mathrm{s}}$ is the flow rate of ASA solution feed in $\mathrm{mL} / \mathrm{min}$, and $c$ is the ASA concentration of the solution in $\mathrm{g} / \mathrm{mL}$.

The process parameters of the continuous crystallization experiments are found in Table 2. The order of the experiments from MSMPR 3 to MSMPR 8 were randomized.

2.2. Methods. 2.2. $\overline{1}$. Determination of the Solubility of ASA. The solubility of the ASA in the reaction mixture was studied in a jacketed glass reactor $(150 \mathrm{~mL}$, Normag, Germany) equipped with a Pt-100 thermometer, a Eurostar power control-visc type stirrer (IKA, Germany), a propeller mixer, and a monofluid thermostat. The speed value of the stirrer was set at $200 \mathrm{rpm}$, and the slurry was tempered at 1,13 , and $25{ }^{\circ} \mathrm{C}$ to measure the solubility in the temperature range of continuous crystallization experiments.

The ASA solubility in the solvent mixture (see in Table 1) was examined as a function of temperature and antisolvent to ASA solution ratio. The conditions of the solubility experiments are detailed in Table 3. Primarily, the exact amounts of the solvent

Table 3. Experimental Conditions of the Solubility Measurements (SE)

\begin{tabular}{ccccc}
$\begin{array}{c}\text { ID of } \\
\text { experiments }\end{array}$ & $\begin{array}{c}\text { volume of the reaction } \\
\text { mixture solvent components } \\
{[\mathrm{mL}]}\end{array}$ & $\begin{array}{c}\text { heptane } \\
{[\mathrm{mL}]}\end{array}$ & $\begin{array}{c}\text { AS/ASA } \\
\text { ratio }[-]\end{array}$ & $\begin{array}{c}\text { ASA } \\
{[\mathrm{g}]}\end{array}$ \\
SE_1 & 70.0 & 0.0 & - & 9.140 \\
SE_2 & 33.0 & 66.0 & $2 / 1$ & 3.003 \\
SE_3 & 20.0 & 80.0 & $4 / 1$ & 2.068 \\
SE_4 & 14.5 & 87.7 & $6 / 1$ & 1.400 \\
\hline
\end{tabular}

mixture and the heptane were poured into the glass reactor and cooled to $1{ }^{\circ} \mathrm{C}$ starting temperature. When the desired temperature was achieved, solid ASA was added to the mixture. The amount of ASA added was more than the expected saturation concentration providing a heterogenic system. An estimation of the amount of solid ASA in the slurry was placed in Supporting Information. The slurry was kept agitated for at least $1 \mathrm{~h}$ to achieve the equilibrium state. After $1 \mathrm{~h}$ of agitation, some volume of the sample was taken and was filtered through a PTFE syringe filter $(0.45 \mu \mathrm{m})$ to reach the saturated ASA solution. These ASA solutions were diluted 100 and 1000 times, dissolved and extracted with a mixture of $\mathrm{ACN}: \mathrm{MeOH}: \mathrm{H}_{3} \mathrm{PO}_{4}(85 \%)$ (92:8:0.5 v/v ratio) due to ensure the chemical stability of ASA $^{32}$ until the HPLC measurement. The concentration of ASA was determined using RP-HPLC. After the first sample, the slurry in the vessel was heated to $13{ }^{\circ} \mathrm{C}$ and sampled after an hour of stirring. This procedure was repeated at $25{ }^{\circ} \mathrm{C}$.

2.2.2. HPLC Analysis. The solubility of ASA and the purity of the product were investigated with an RP-HPLC (Agilent 1200 series LC System) according to the method that was used to determine the purity of the ASA reaction mixture after the synthesis by Balogh and his co-workers. ${ }^{6}$ During this process, a $5 \mu \mathrm{L}$ sample volume was injected onto a Supelco Inertsil ODS-2 C18 column $(5 \mu \mathrm{m} ; 250 \times 4.6$ $\mathrm{mm}$ ). Isocratic elution was performed using an eluent containing $60 \%$

\section{Table 2. Experimental Conditions of Continuous Crystallization Experiments}

\begin{tabular}{|c|c|c|c|c|c|}
\hline ID of experiment & $\begin{array}{c}\text { temperature } \\
{\left[{ }^{\circ} \mathrm{C}\right]}\end{array}$ & ASA solution feed flow rate $[\mathrm{mL} / \mathrm{min}]$ & heptane feed flow rate $[\mathrm{mL} / \mathrm{min}]$ & residence time $[\mathrm{min}]$ & overall time of exp. $[\mathrm{h}]$ \\
\hline MSMPR_1 & 12.0 & 2 & 8 & 23.50 & 4.09 \\
\hline MSMPR_2 & 25.0 & 2 & 8 & 23.50 & 4.29 \\
\hline MSMPR_3 & 2.5 & 4 & 16 & 11.75 & 4.00 \\
\hline MSMPR_4 & 2.5 & 2 & 8 & 23.50 & 7.95 \\
\hline MSMPR_5 & 2.5 & 1 & 4 & 47.00 & 15.93 \\
\hline MSMPR_6 & 25.0 & 4 & 16 & 11.75 & 4.00 \\
\hline MSMPR_7 & 25.0 & 2 & 8 & 23.50 & 7.95 \\
\hline MSMPR_8 & 25.0 & 1 & 4 & 47.00 & 16.02 \\
\hline
\end{tabular}


ACN and 40\% water-phosphoric acid mixture (200:1 ratio). The measurement lasts $4 \mathrm{~min}$; the retention time of ASA and SA is at 2.5 and $3.1 \mathrm{~min}$, respectively. The exact ASA concentration measurement was based on the sum of the ASA and the impurity SA peak areas. The impurity content of the crystalline product was determined based on the ratio of the peak areas.

2.2.3. Particle Size Distribution and Visual Measurement. The particle size distribution (PSD) was measured offline using a Parsum IPP 70-s inline particle sizing probe in batch mode. The samples were dispersed into the probe for estimating the cord length of the crystals. The volumetric distribution values $D_{\mathrm{v}} 10, D_{\mathrm{v}} 50$, and $D_{\mathrm{v}} 90$ were applied to characterize the crystal size distribution (CSD) of the products. The CSD plots represent volume-based distributions where crystal size intervals are plotted as a function of the volume fraction (\%). The volume fraction (\%) of $x_{\mathrm{i}}$ crystal size interval represents the volume percent of these crystals relative to the total crystal fraction. The crystal habit of the products was monitored by using a CKX 53 inverse microscope equipped with an 18Mp CAM-SC180 Camera set.

\section{RESULTS AND DISCUSSION}

3.1. Solubility Measurement. Based on the results of the preliminary batch experiments, heptane was chosen as an effective antisolvent for ASA crystallization. In further batch experiments, the effect of antisolvent to ASA solution ratio, residence time, and temperature on the yield and product purity were studied. Details of these batch experiments are in the Supporting Information.

The solubility of the ASA in the exact composition of the reaction mixture and also in the presence of heptane antisolvent was measured at three temperatures (1, 13, 25 $\left.{ }^{\circ} \mathrm{C}\right)$. The determined saturation concentrations are presented in Figure 2.

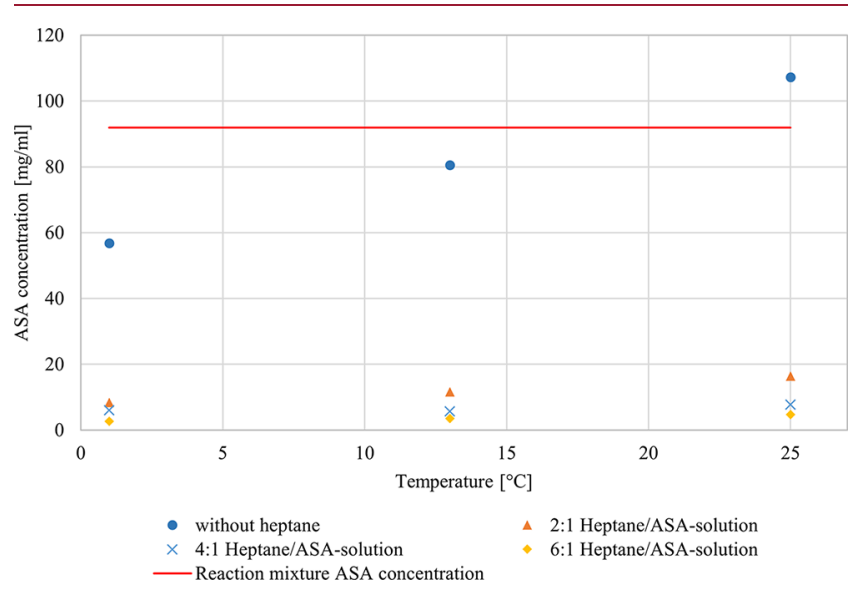

Figure 2. Solubility of ASA in the reaction mixture without heptane, with $2: 1,4: 1$, and $6: 1$ heptane to ASA solution $\mathrm{v} / \mathrm{v}$ ratio. The concentration of ASA in the reaction mixture (red line, $91.9 \mathrm{mg} / \mathrm{mL}$ ).

The blue points represent the saturation of ASA in the solvent composition of the pure reaction mixture. This saturation curve indicates a slight dependence of solubility as a function of temperature; thus, cooling itself is not enough for reaching reasonable yield. The red line represents the ASA concentration in the initial reaction mixture $(91.9 \mathrm{mg} / \mathrm{mL})$, which is a near-saturated solution based on the saturation data at room temperature. The orange, green, and purple points represent the 2:1, 4:1, and 6:1 heptane to ASA solution v/v ratio, consecutively. The results highlight that the antisolvent significantly affects the solubility of ASA in the reaction mixture. Moreover, the increasing amount of antisolvent results in higher supersaturation, which leads to better productivity as well. Considering the productivity and economic aspects, antisolvent and combined cooling/antisolvent crystallization were implemented at 4:1 heptane to ASA solution ratio.

3.2. Position of the Buffer Element. A vertical plate as a buffer element was placed in the reactor to ensure homogeneous slurry withdrawal. The schematic images of the applied buffer element positions are illustrated in Figure 3.

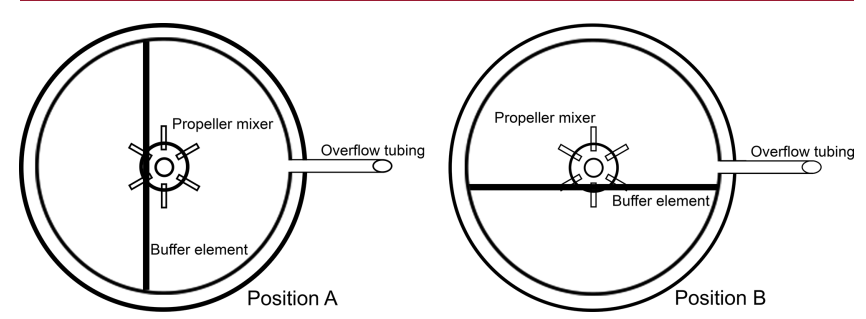

Figure 3. Schematic upward images of buffer element position $\mathrm{A}$ and position B.

The position of the vertical plate affects the efficacy of product withdrawal. By appling position A, the number of crystals in the withdrawn slurry was representative at the beginning of the experiment, whereas later the quantity of the discharged crystals was not sufficient. During the start-up phase, the density in the reactor tends to increase, while the yield is decreasing. The explanation for this phenomenon is that the smaller crystals produced in the beginning are removable easily. However, growing crystals are more likely to cause sedimentation problems, so ensuring efficient mixing conditions is essential. In MSMPR_1, the steady-state conditions were not achieved, as the product withdrawal was not representative. The amount and the quality of removed crystals were not the same as the solids agitated in the reactor. In contrast, by utilizing position $\mathrm{B}$, the yield remains almost constant (see in Figure 4). Consequently, we used position B for further continuous crystallization to provide representative product removal.
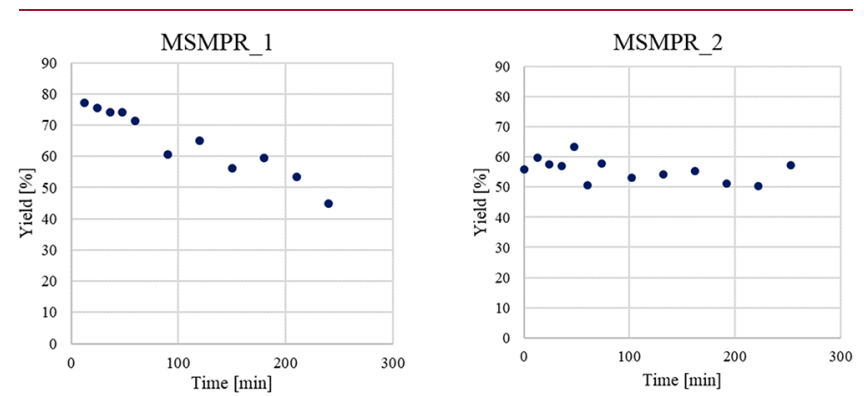

Figure 4. Yield during MSMPR 1 (position A) and MSMPR 2 (position B).

It was observed that the product quality similar in the absence of the buffer element as well. Thus, the applied buffer element did not affect the crystallization process significantly. Additionally, the buffer element was necessary for representative product removal.

3.3. Evaluation of the Product Quality. 3.3.1. Characterization of the Product Purity, the Process Yield, and Productivity. A series of experiments has been conducted to investigate the changes in the product features (i.e., purity, crystal size distribution, crystal habit) and process productivity 
by varying the process parameters. In the following section, the results of the process characterization will be detailed.

The onset of steady-state operation was detected by analyzing the crystal morphology, namely, the crystal habit, crystal size, and CSD. The steady-state operation could be observed after the crystallization procedure has already lasted for six to ten residence times in each of the experiments independently of the adjusted process parameters. After reaching the steady-state operation, stable product quality and quantity were achieved. The removed product was representative, since the crystal size, CSD, and crystal habit of the sampling and the reactor content were determined straight after the sampling proved to be the same.

In terms of purity, the SA content was less than $0.81 \%$ in each experiment. The average impurity of the products was $0.30 \pm 0.16 \%$ evidenced by HPLC measurement; thus, the developed direct processing technique is proper for cleaning the reaction mixture from undesired impurities. Meeting the $3 \%$ regulatory limit is achievable when this technique is used. In Figure 5, it is shown that in the examined design space the

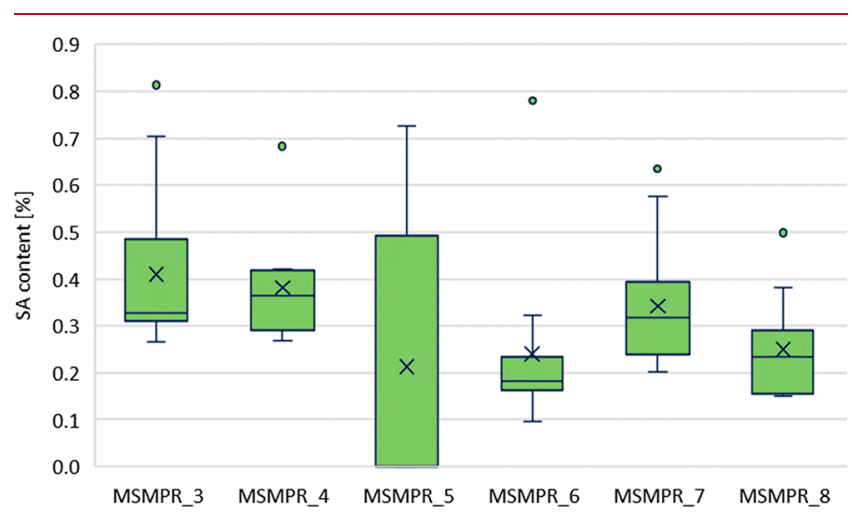

Figure 5. Box-plot diagram of SA impurity content of the products.

process parameters do not affect the dispersion of the impurity content significantly. It can be assumed that SA remains dissolved in the mother liquor. As the filter cake was not washed, the source of the product SA content could be the mother liquor adhered to the crystal surface. To decrease SA concentration, an adequate washing procedure could be developed for this system to eliminate the adhered mother liquor from the surface of the filter cake.

The continuous crystallization experiments were characterized by yield and productivity. We calculated the yield of the samplings $\left(y_{\mathrm{s}, X}, \%\right)$ from the weight of dry crystalline product samples $\left(m_{\mathrm{s}}, \mathrm{g}\right)$ and the fed ASA amount $\left(m_{\mathrm{f}}, \mathrm{g}\right)$ estimated from the length of the sampling $\left(t_{\mathrm{f}}\right)$, ASA solution flow rate $\left(\dot{V}_{s}\right)$, and concentration $(c)$ (eq 1$)$.

$$
y_{\mathrm{s}, X}=\frac{m_{\mathrm{s}}}{m_{\mathrm{f}}} \times 100
$$

After placing the inner vertical plate in position B, almost constant yields were obtained from the beginning of the experiments. Hence, each experiment could be featured by the average yield $\left(y_{\mathrm{Av}}, \%\right)$ and its standard deviation (St. dev. \%) determined from values of sample yields (eqs 3 and 4).

$$
y_{\mathrm{Av}}=\frac{\sum y_{\mathrm{s}, X}}{n} \times 100
$$

$$
\text { St. dev. }=\sqrt{\frac{\sum\left(y_{\mathrm{s}, X}-\bar{y}\right)^{2}}{(n-1)}}
$$

where $n$ is the number of samples [-], and $\bar{y}$ is the mean value of the yield in \%.

The values of yield and process productivity can be shown in Table 4.

Table 4. Summary of the MSMPR Crystallization Experiments Including Process Parameters, Yield, and Productivity

\begin{tabular}{lrccc}
$\begin{array}{c}\text { ID of the } \\
\text { experiment }\end{array}$ & $\begin{array}{c}\text { temperature } \\
{\left[{ }^{\circ} \mathrm{C}\right]}\end{array}$ & $\begin{array}{c}\text { residence } \\
\text { time [min] }\end{array}$ & yield [\%] & $\begin{array}{c}\text { productivity } \\
{[\mathrm{g} / \mathrm{h}]}\end{array}$ \\
\hline MSMPR_3 & 2.5 & 11.75 & $78.6 \pm 2.8$ & 17.3 \\
MSMPR_4 & 2.5 & 23.50 & $74.1 \pm 3.2$ & 8.2 \\
MSMPR_5 & 2.5 & 47.00 & $76.8 \pm 3.7$ & 4.2 \\
MSMPR_6 & 25.0 & 11.75 & $59.2 \pm 2.7$ & 13.1 \\
MSMPR_7 & 25.0 & 23.50 & $64.4 \pm 6.1$ & 7.1 \\
MSMPR_8 & 25.0 & 47.00 & $57.6 \pm 5.8$ & 3.2 \\
\hline
\end{tabular}

As can be seen in Table 4, lower temperature resulted in a significant increase in yield, while residence time had no remarkable effect on the product quantity. In most of the experiments, the standard deviation of yield remained under $4 \%$. The highest standard deviation regarding yield was $6 \%$ in the MSMPR_7 ( T: $\left.25^{\circ} \mathrm{C}, \mathrm{RT}: 23.5 \mathrm{~min}\right)$ experiment.

It is important to mention that several conditions could potentially alter the yield by applying an overflow and vertical plate. Some examples are as follows: (1) the variation in feed flow rate caused by the inefficiently operated pumps; (2) the deposition and the detachment of the crystals on/from the reactor wall or on/from the surface of the vertical plate; (3) the position of feeding tubes. If these tubes were placed close to the wall or each other, it could result in local inhomogeneity in concentration intensifying the deposition mechanisms. We experienced these events during the process development and made an effort to eliminate all these contingencies in the presented results.

The productivity of the experiments varied in the range of $3.2-17.3 \mathrm{~g} / \mathrm{h}$. Shorter residence time and lower temperatures favor an increase in productivity. Hence, the highest productivity could be obtained when the residence time is shorter $(11.75 \mathrm{~min})$, but the yield is high as a result of the lower operating temperature $(78.6 \pm 2.8 \%)$.

3.3.2. Alteration of Crystal Morphology. To monitor the main tendency in crystal morphology changes during MSMPR crystallizations, two processes are demonstrated. All process parameters of the selected experiments are different, as it can illustrate the difference caused by varying operating circumstances. In Table 5, Table 6, and Table 7, two selected crystallizations are described by evaluating the samples with $D_{\mathrm{v}} 10, D_{\mathrm{v}} 50, D_{\mathrm{v}} 90$, and $D[4,3]$ values, boxplot diagrams, microscopic pictures, and particle size distribution. The volume mean diameters $(D[4,3])$, presented besides $D_{\mathrm{v}}$ values, are generally suitable for the description of polydisperse systems. In this case, the volume mean diameter was similar to $D_{\mathrm{v}} 50$.

The ASA solution and antisolvent were fed into the initial suspension; thus, secondary nucleation occurred during the continuous process. After one residence time, the product crystals were significantly smaller and less columnar at low temperature $\left(2.5{ }^{\circ} \mathrm{C}, D_{\mathrm{v}} 50: 306 \mu \mathrm{m}\right)$, while at room 
Table 5. Comparison of MSMPR $3\left(2.5^{\circ} \mathrm{C}, 11.75 \mathrm{~min}\right)$ and MSMPR_8 $\left(25^{\circ} \mathrm{C}, 47 \mathrm{~min}\right)$ Experiment Regarding Crystal Size Alteration during the Crystallization Process

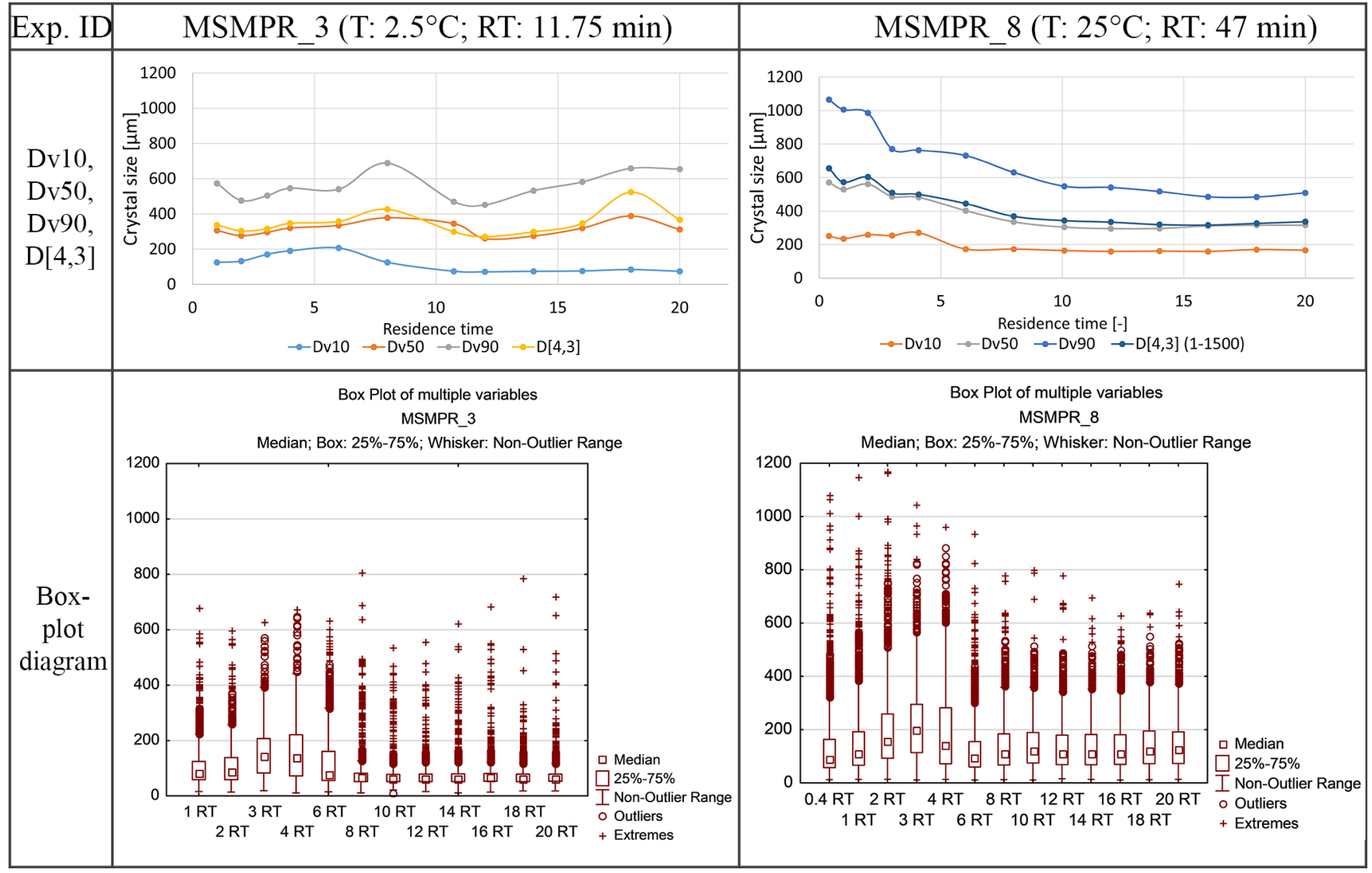

Table 6. Microscopic Pictures (4X), $D_{v}$ Values, and CSD Plots of the MSMPR_3 Samples (1 RT, 10 RT, and from the Reactor at the End of the Experiment)

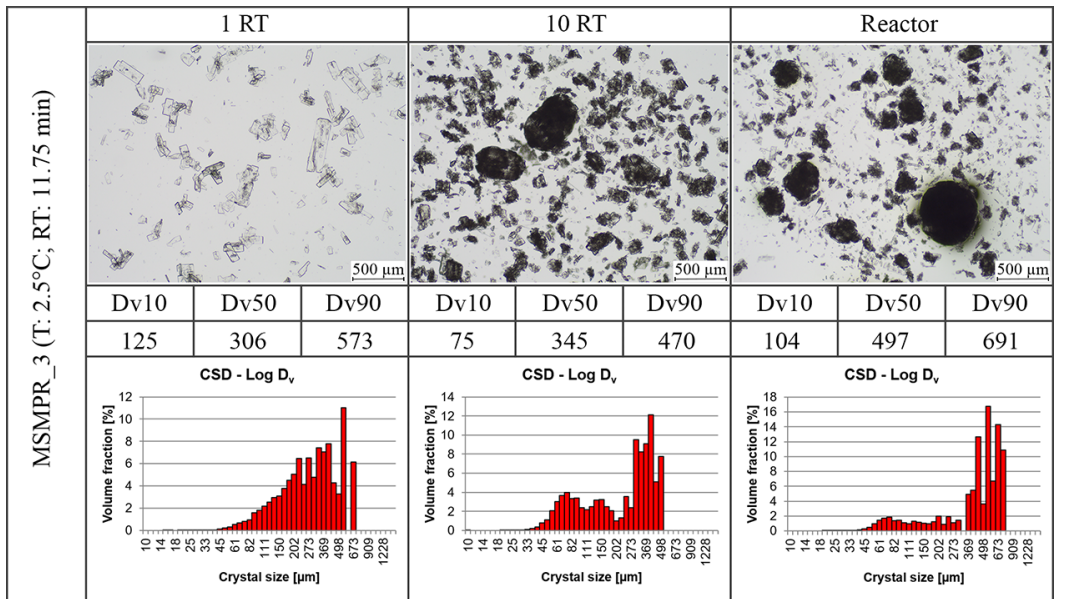

temperature the product contained large $\left(D_{\mathrm{v}} 50: 707 \mu \mathrm{m}\right)$ columnar crystals that were growing in all directions. During the first phase of both experiments, the crystals were growing and the CSDs were broadening. Before the onset of steadystate operation, reaching the critical size for more than five and seven residence times, the crystals started to fragment. This led to an increase in the amount of smaller crystal fraction. This mechanism could be monitored on the microscopic pictures and the boxplot graphics at both studied temperatures. The steady-state conditions were reached in both processes after more than eight residence times. Most of the large columnar particles were transformed into spherical and isometric crystals. At low operation temperature, the product was polydisperse with two characteristic crystal fractions, while at room temperature, the manufactured crystals were unimodal, according to the volumetric distributions.

A similar process was identified by examining the boxplot diagram. Smaller crystals and narrower CSD were established at lower temperatures. The product size and CSD were growing and broadening for a certain crystal size when 
Table 7. Microscopic Pictures $(4 \times), D_{v}$ Values, and CSD Plots of the MSMPR_8 Samples (1 RT, 10 RT, and from the Reactor at the End of the Experiment)

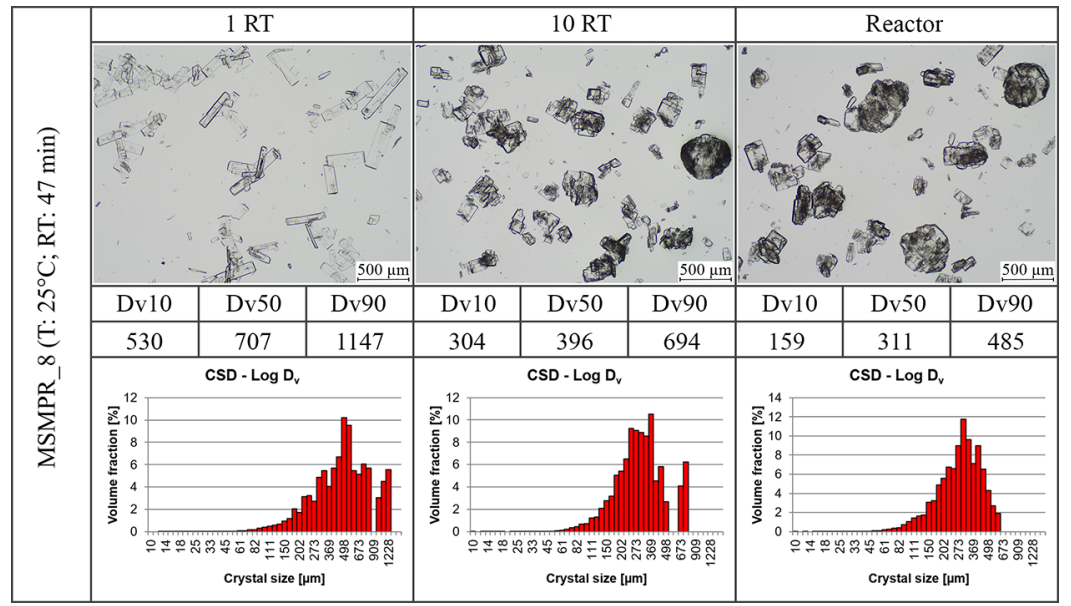

Table 8. Summary of the MSMPR Crystallization Experiments at $2.5^{\circ} \mathrm{C}$ Regarding Crystal Habit, Crystal Size, and Crystal Size Distribution of the Experiments under Steady-State Operation

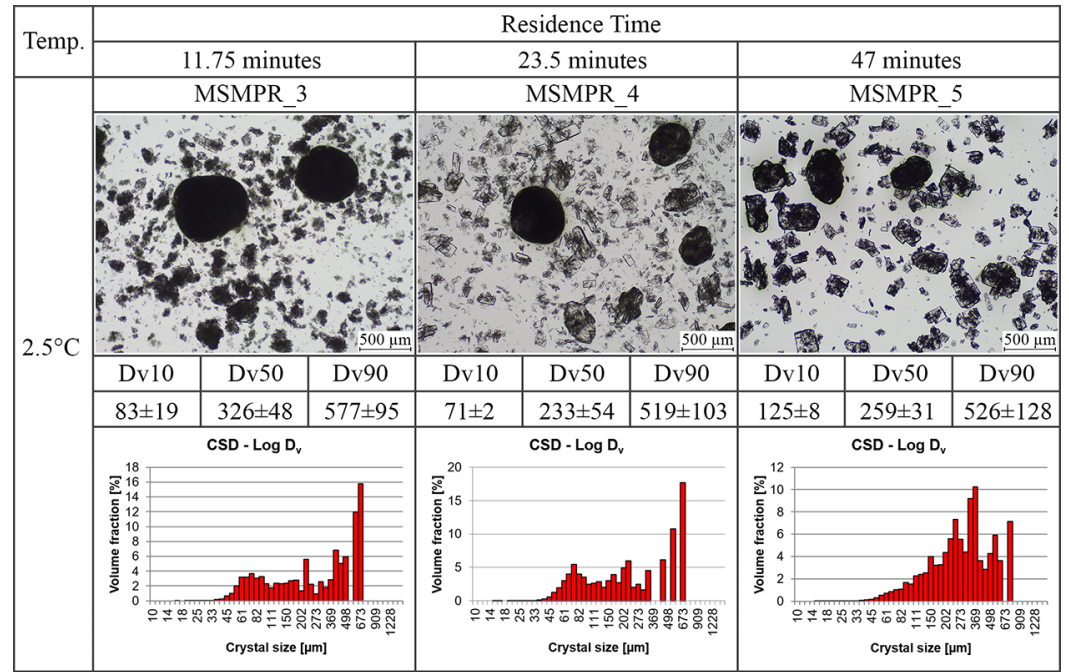

Table 9. Summary of the MSMPR Crystallization Experiments at $25^{\circ} \mathrm{C}$ Regarding Crystal Habit, Crystal Size, and Crystal Size Distribution of the Experiments under Steady-State Operation

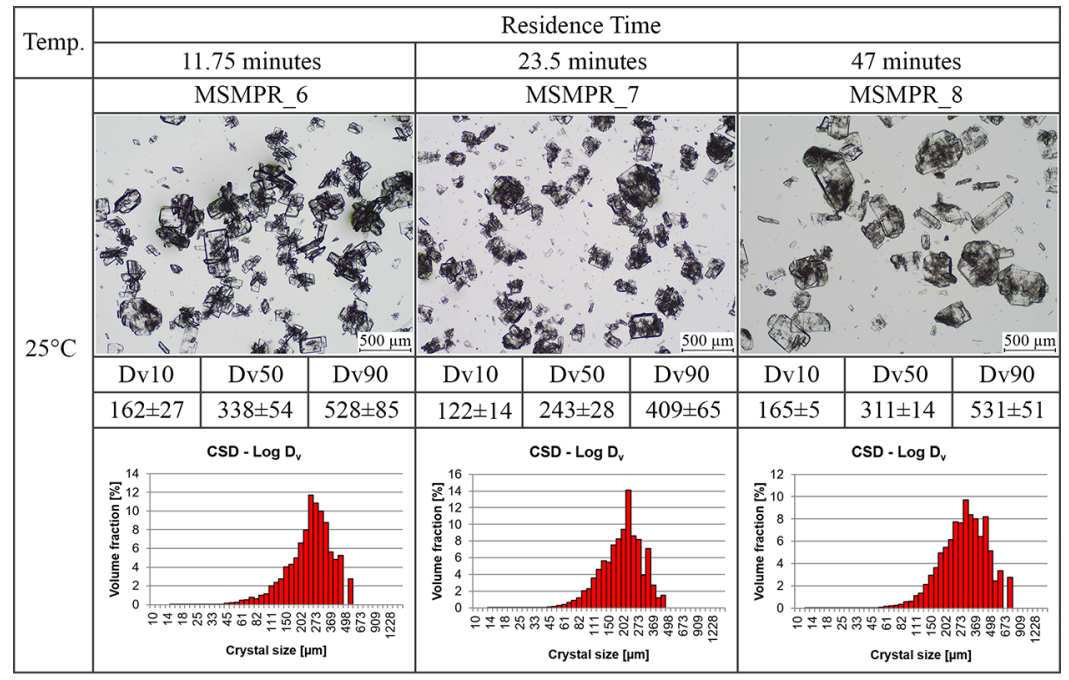


disintegration became dominant. When the temperature was set at $2.5{ }^{\circ} \mathrm{C}$ the majority of the particles were small; however, a great amount of significantly larger crystals (outliers and extremes $>75 \%$ ) were detected in steady-state operation. At room temperature, in contrast, the distributions of the outliers and extremes were smaller.

3.3.3. Particle Size Distribution. In Table 8 and Table 9, the microscopic images, the average values, and the standard deviation of $D_{\mathrm{v}} 10, D_{\mathrm{v}} 50$, and $D_{\mathrm{v}} 90$, and the volume density distributions of the output are presented to illustrate the effect of studied temperatures and residence times on CSD. At 2.5 ${ }^{\circ} \mathrm{C}$, the supersaturation was higher compared to higher operation temperatures, and thus nucleation dominated during the crystallization process resulting in smaller crystals. As already established, a few large isometric crystals were identified at the beginning of the process. These crystals could be either grown and resulted in crystals with rounded surface or aggregates bounding smaller particles on the surface with time. At $2.5^{\circ} \mathrm{C}$, large size differences could be observed among the produced crystal fractions as a function of decreasing residence time, which accelerated the particle sedimentation. As a result, the products were polydisperse with a broad CSD curve, especially when shorter residence time was set $\left(D_{\mathrm{v}} 10: 83 \pm 19 \mu \mathrm{m} ; D_{\mathrm{v}} 90: 577 \pm 95 \mu \mathrm{m}\right)$. In contrast, lower supersaturation could be achieved at room temperature. Hence, the crystal growth rate could be higher, resulting in wide unimodal crystal size distribution. It was found that the length of the residence time does not change the quality of the crystalline product significantly in the investigated residence time range. Consequently, it was evidenced that the main differentiator was the temperature, as the residence time effect was not significant.

The following steps of processing the large, isometric, and uniform particles with good flowability are preferred for the pharmaceutical formulation. In contrast, polydispersity in the product leads to the separation of fractions that is undesired in tableting steps. Previous investigations of the starting material by the authors confirmed that the presence of needle-shaped or columnar crystals, smaller than $75 \mu \mathrm{m}$, worsens the flowability of particles below the level needed for direct compression. Further details of this investigation are presented in the Supporting Information. Accordingly, the ratio of this fraction was investigated and shown in Figure 6 . At $2.5^{\circ} \mathrm{C}$, the amount of these small crystals increased up to $12 \mathrm{v} / \mathrm{v} \%$. At $25{ }^{\circ} \mathrm{C}$, as low as $0.5 \mathrm{v} / \mathrm{v} \%$ small-sized fractions could be achieved, which assists better flowability. Consequently, longer residence time

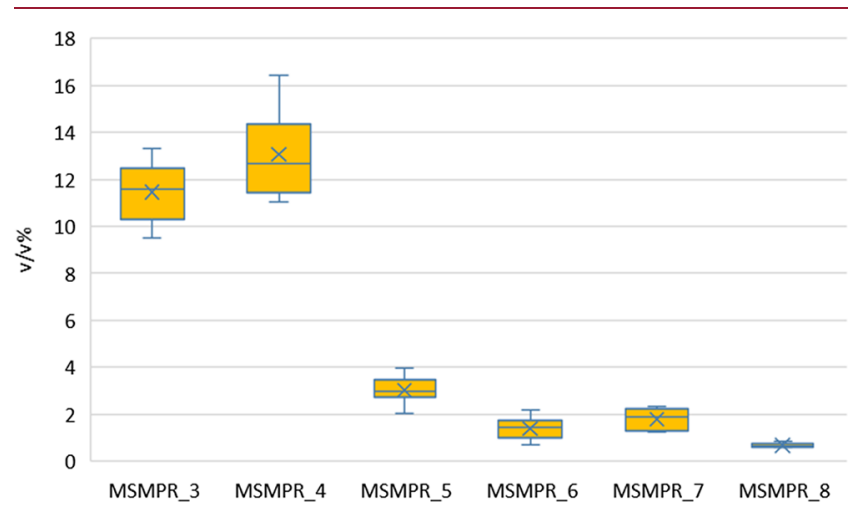

Figure 6. V/V\% of particles sized $75 \mu \mathrm{m}$ or smaller. and higher temperatures provide better product quality in the aspect of the formulation procedure of the crystals.

\section{CONCLUSION}

Most of the published continuous crystallization studies focus on the crystallization of a product from pure drug solution. We developed, in contrast, the continuous crystallization of a complex, multicomponent reaction mixture. For this purpose, MSMPR crystallizer was equipped with an overflow tube for product removal and an inner vertical plate as a buffer element to prevent the immediate elimination of the fed materials and also ensure the representative withdrawal of the produced polydisperse crystals. The formation of polydisperse products was also observed in the absence of the buffer element. The buffer element was necessary for representative product removal of the produced polydisperse crystals.

The effect of the position of the buffer element on product withdrawal efficacy was investigated. It was found that the position parallel to the outlet tubing supports constant crystal withdrawal independently of crystal size and CSD. It has been proven that using pump-free solutions like overflow piper or tubes for product withdrawal could be an effective alternative of conventional peristaltic pumps. Further advantages of using this technology are that the difficulties of level control and plugging issues during product removal could be eliminated. System operations are more cost-effective, since fewer pumps are required for material transfer leading to lower investment and operational costs.

Furthermore, the effect of temperature and length of residence time on product quality (purity, crystal habit, and CSD) and quantity was examined. The significant impact of temperature on yield, crystal size, and CSD has been detected, but no effect on product impurity content was found. Consequently, yield could be increased by decreasing the operating temperature. Furthermore, the average SA impurity content was $0.3 \%$, far below the $3 \%$ regulatory limit. The low temperature $\left(2.5{ }^{\circ} \mathrm{C}\right)$ favored the generation of a smaller crystal fraction due to higher supersaturation. Polydisperse products could be characterized by a wide and multimodal CSD. By appling higher temperatures $\left(25^{\circ} \mathrm{C}\right)$, large crystals with unimodal distribution could be produced. The length of the residence time had no significant effect on the examined product parameters in the investigated residence time range. Additionally, in accordance with the preliminary batch experiments presented in the Supporting Information, the kinetics of the investigated crystallization system was rather fast. Due to the fast kinetics of this system, the tubular crystallizer could be suitable for crystallization as well.

In conclusion, separation and purification of ASA from a multicomponent reaction mixture could be performed based on the developed continuous crystallization process utilizing a single-stage MSMPR crystallizer. Investigating the relation between the process parameters and the product quality, we found that by increasing the operating temperature to $25{ }^{\circ} \mathrm{C}$ the obtained particles of unimodal crystal size distribution could be more adequate for the subsequent formulation steps of the crystalline product. Therefore, the developed continuous crystallization method can be integrated well into the end-toend production chain. 


\section{ASSOCIATED CONTENT}

\section{SI Supporting Information}

The Supporting Information is available free of charge at https://pubs.acs.org/doi/10.1021/acs.cgd.0c00252.

Further details about antisolvent selection, preliminary batch experiments, solubility, and flowability measurements (PDF)

\section{AUTHOR INFORMATION}

\section{Corresponding Author}

Hajnalka Pataki - Budapest University of Technology, Department of Organic Chemistry and Technology, Budapest 1111, Hungary; orcid.org/0000-0002-8103-0601; Email: patakihajni@gmail.com

\section{Authors}

Kornélia Tacsi - Budapest University of Technology, Department of Organic Chemistry and Technology, Budapest 1111, Hungary; 이이. orcid.org/0000-0001-8506-5062

András Domokos - Budapest University of Technology, Department of Organic Chemistry and Technology, Budapest 1111, Hungary

Brigitta Nagy - Budapest University of Technology, Department of Organic Chemistry and Technology, Budapest 1111, Hungary

István Csontos - Budapest University of Technology, Department of Organic Chemistry and Technology, Budapest 1111, Hungary

Imre Markovits - Egis Pharmaceuticals PLC, Drug Substance Pilot Plant, Drug Substance Development Division, Directorate of Drug Substance Development, Budapest 1106, Hungary

Ferenc Farkas - Egis Pharmaceuticals PLC, Drug Substance Pilot Plant, Drug Substance Development Division, Directorate of Drug Substance Development, Budapest 1106, Hungary

Zsombor Kristóf Nagy - Budapest University of Technology, Department of Organic Chemistry and Technology, Budapest 1111, Hungary; $\odot$ orcid.org/0000-0003-2651-7756

György Marosi - Budapest University of Technology, Department of Organic Chemistry and Technology, Budapest 1111, Hungary

Complete contact information is available at:

https://pubs.acs.org/10.1021/acs.cgd.0c00252

\section{Notes}

The authors declare no competing financial interest.

\section{ACKNOWLEDGMENTS}

This research was financially supported by the Hungarian Scientific Research Fund (OTKA PD121143, FK-132133, KH129584). FIEK_16-1-2016-0007 has been implemented with the support provided from the National Research, Development and Innovation Fund of Hungary, financed under the Centre for Higher Education and Industrial Cooperation Research infrastructure development (FIEK 16) funding scheme. Hajnalka Pataki is thankful for the János Bolyai Research Scholarship of the Hungarian Academy of Sciences. B. Nagy was supported by the scholarship of the Rosztoczy Foundation. The research was supported by the UNKP-19-3 New National Excellence Program of the Ministry of Human Capacities.

\section{REFERENCES}

(1) Lee, S. L.; O'Connor, T. F.; Yang, X.; Cruz, C. N.; Chatterjee, S.; Madurawe, R. D.; Moore, C. M. V.; Yu, L. X.; Woodcock, J. Modernizing Pharmaceutical Manufacturing: From Batch to Continuous Production. J. Pharm. Innov. 2015, 10, 191-199.

(2) Poechlauer, P.; Colberg, J.; Fisher, E.; Jansen, M.; Johnson, M. D.; Koenig, S. G.; Lawler, M.; Laporte, T.; Manley, J.; Martin, B.; O'Kearney-McMullan, A. Pharmaceutical Roundtable Study Demonstrates the Value of Continuous Manufacturing in the Design of Greener Processes. Org. Process Res. Dev. 2013, 17, 1472-1478.

(3) Lawton, S.; Steele, G.; Shering, P.; Zhao, L.; Laird, I.; Ni, X. W. Continuous Crystallization of Pharmaceuticals Using a Continuous Oscillatory Baffled Crystallizer. Org. Process Res. Dev. 2009, 13, 13571363.

(4) Quon, J. L.; Zhang, H.; Alvarez, A.; Evans, J.; Myerson, A. S.; Trout, B. L. Continuous Crystallization of Aliskiren Hemifumarate. Cryst. Growth Des. 2012, 12, 3036-3044.

(5) Mascia, S.; Heider, P. L.; Zhang, H.; Lakerveld, R.; Benyahia, B.; Barton, P. I.; Braatz, R. D.; Cooney, C. L.; Evans, J. M. B.; Jamison, T. F.; Jensen, K. F.; Myerson, A. S.; Trout, B. L. End-to-End Continuous Manufacturing of Pharmaceuticals: Integrated Synthesis, Purification, and Final Dosage Formation. Angew. Chem., Int. Ed. 2013, 52, 1235912363.

(6) Balogh, A.; Domokos, A.; Farkas, B.; Farkas, A.; Rapi, Z.; Kiss, D.; Nyiri, Z.; Eke, Z.; Szarka, G.; Örkényi, R.; Mátravölgyi, B.; Faigl, F.; Marosi, G.; Nagy, Z. K. Continuous End-to-End Production of Solid Drug Dosage Forms: Coupling Flow Synthesis and Formulation by Electrospinning. Chem. Eng. J. 2018, 350, 290-299.

(7) Chen, J.; Sarma, B.; Evans, J. M. B.; Myerson, A. S. Pharmaceutical Crystallization. Cryst. Growth Des. 2011, 11, 887-895.

(8) Hou, G.; Power, G.; Barrett, M.; Glennon, B.; Morris, G.; Zhao, Y. Development and Characterization of a Single Stage MixedSuspension, Mixed-Product-Removal Crystallization Process with a Novel Transfer Unit. Cryst. Growth Des. 2014, 14, 1782-1793.

(9) Randolph, A. D.; Larson, M. A. Transient and Steady State Size Distributions in Continuous Mixed Suspension Crystallizers. AIChE J. 1962, 8, 639-645.

(10) Randolph, A. D.; Larson, M. A. Theory of Particulate ProcessesAnalysis and Techniques of Continuous Crystallization; 1971, p 64. .

(11) Alvarez, A. J.; Myerson, A. S. Continuous Plug Flow Crystallization of Pharmaceutical Compounds. Cryst. Growth Des. 2010, 10, 2219-2228.

(12) Jiang, M.; Braatz, R. D. Designs of Continuous-Flow Pharmaceutical Crystallizers: Developments and Practice. CrystEngComm 2019, 21, 3534-3551.

(13) Brown, C.; McGlone, T.; Florence, A. Continuous Crystallisation. Contin. Manuf. Pharm. 2017, 169-226.

(14) Alvarez, A. J.; Singh, A.; Myerson, A. S. Crystallization of Cyclosporine in a Multistage Continuous MSMPR Crystallizer. Cryst. Growth Des. 2011, 11, 4392-4400.

(15) Zhang, H.; Quon, J.; Alvarez, A. J.; Evans, J.; Myerson, A. S.; Trout, B. Development of Continuous Anti-Solvent/Cooling Crystallization Process Using Cascaded Mixed Suspension, Mixed Product Removal Crystallizers. Org. Process Res. Dev. 2012, 16, 915924

(16) Zhang, D.; Xu, S.; Du, S.; Wang, J.; Gong, J. Progress of Pharmaceutical Continuous Crystallization. Engineering 2017, 3, 354364.

(17) Sha, L.; Hatakka, H.; Louhi, M.; Palosaari, S. Crystallization Kinetics of Potassium Sulfate in an MSMPR Stirred Crystallizer. J. Cryst. Growth 1996, 166, 1105-1110.

(18) Ferguson, S.; Morris, G.; Hao, H.; Barrett, M.; Glennon, B. Characterization of the Anti-Solvent Batch, Plug Flow and MSMPR Crystallization of Benzoic Acid. Chem. Eng. Sci. 2013, 104, 44-54.

(19) Schall, J. M.; Mandur, J. S.; Braatz, R. D.; Myerson, A. S. Nucleation and Growth Kinetics for Combined Cooling and Antisolvent Crystallization in an MSMPR System - Estimating Solvent Dependency. Cryst. Growth Des. 2018, 18, 1560-1570. 
(20) Tahara, K.; Kono, Y.; Myerson, A. S.; Takeuchi, H. Development of Continuous Spherical Crystallization to Prepare Fenofibrate Agglomerates with Impurity Complexation Using MSMPR Crystallizer. Cryst. Growth Des. 2018, 18, 6448-6454.

(21) Powell, K. A.; Saleemi, A. N.; Rielly, C. D.; Nagy, Z. K. Periodic Steady-State Flow Crystallization of a Pharmaceutical Drug Using MSMPR Operation. Chem. Eng. Process. 2015, 97, 195-212.

(22) Li, J.; Trout, B. L.; Myerson, A. S. Multistage Continuous Mixed-Suspension, Mixed-Product Removal (MSMPR) Crystallization with Solids Recycle. Org. Process Res. Dev. 2016, 20, 510-516.

(23) Ferguson, S.; Ortner, F.; Quon, J.; Peeva, L.; Livingston, A.; Trout, B. L.; Myerson, A. S. Use of Continuous MSMPR Crystallization with Integrated Nanofiltration Membrane Recycle for Enhanced Yield and Purity in API Crystallization. Cryst. Growth Des. 2014, 14, 617-627.

(24) Onyemelukwe, I. I.; Parsons, A. R.; Wheatcroft, H. P.; Robertson, A.; Nagy, Z. K.; Rielly, C. D. The Role of Residence Time Distribution in the Continuous Steady-State Mixed Suspension Mixed Product Removal Crystallization of Glycine. Cryst. Growth Des. 2019, $19,66-80$.

(25) Lührmann, M.-C.; Timmermann, J.; Schembecker, G.; Wohlgemuth, K. Enhanced Product Quality Control through Separation of Crystallization Phenomena in a Four-Stage MSMPR Cascade Enhanced Product Quality Control through Separation of Crystallization Phenomena in a Four-Stage MSMPR Cascade. Cryst. Growth Des. 2018, 18, 7323-7334.

(26) Narducci, O.; Jones, A. G.; Kougoulos, E. Continuous Crystallization of Adipic Acid with Ultrasound. Chem. Eng. Sci. 2011, 66, 1069-1076.

(27) Aeschbach, S.; Bourne, J. R. The Attainment of Homogeneous Suspension in a Continuous Stirred Tank. Chem. Eng. J. 1972, 4, 234-242.

(28) Pal, S.; Madane, K.; Kulkarni, A. A. Antisolvent Based Precipitation: Batch, Capillary Flow Reactor and Impinging Jet Reactor. Chem. Eng. J. 2019, 369, 1161-1171.

(29) Besenhard, M. O.; Neugebauer, P.; Scheibelhofer, O.; Khinast, J. G. Crystal Engineering in Continuous Plug-Flow Crystallizers. Cryst. Growth Des. 2017, 17, 6432-6444.

(30) Adamo, A.; Beingessner, R. L.; Behnam, M.; Chen, J.; Jamison, T. F.; Jensen, K. F.; Monbaliu, J.-C. M.; Myerson, A. S.; Revalor, E. M.; Snead, D. R.; Stelzer, T.; Weeranoppanant, N.; Wong, S. Y.; Zhang, P. On-Demand Continuous-Flow Production of Pharmaceuticals in a Compact, Reconfigurable System. Science (Washington, DC, U. S.) 2016, 352, 61-68.

(31) Ricardo, C.; Xiongwei, N. Evaluation and Establishment of a Cleaning Protocol for the Production of Vanisal Sodium and Aspirin Using a Continuous Oscillatory Baffled Reactor. Org. Process Res. Dev. 2009, 13, 1080-1087.

(32) Fogel, J.; Epstein, P.; Chen, P. Simultaneous High-Performance Liquid Chromatography Assay of Acetylsalicylic Acid and Salicylic Acid in Film- Coated Aspirin Tablets. J. Chromatogr. A 1984, 317, 507-511. 